\title{
PERSPECTIVE
}

\section{Ophthalmology in the post-genomic era}

\author{
G C M Black, M E Boulton, P N Bishop, D McLeod
}

The year is 2019 .

Nature Genetics is no more, the genome project a distant memory. The heady days of cloning, sequencing, positional cloning, and candidate gene approaches are long forgotten.

The disease genes are now conquered.

While molecular biologists and geneticists gather before the job centre, their polymerase chain reaction machines are strewn in ever amplifying heaps behind now defunct molecular institutes. The phrase "an interest in ocular genetics" is now treated with derision where once there was only awed incomprehension: surgeons are restored to their position atop the ophthalmological hierarchy.

And still the patients come, ever aging, with their cataracts and their glaucoma, their maculae wet and dry. The production lines of phakos, trabs, and BD8s grind on and on.

An unlikely fantasy or a reasonable prediction?

We have learned, but have we moved forward? For all the wonders of the genomic race, for all the millions spent cloning disease genes, the clinical practice of ophthalmology has yet to alter radically. But what of the next 20 years? What will be the next scientific advances? More importantly how will they impact upon our patients? Who will fill the institutes and laboratories in 20 years' time and, more pertinently for worried molecular biologists, what skills will they need? Whence the post-genomic scientists?

In recent times we have, perhaps inevitably, seen a somewhat reductionist focus on single genes and the Mendelian disorders they underlie. Our understanding of a wide range of disorders, affecting probably every ocular structure, has been enhanced by novel and often utterly unexpected insights into their causation and classification. We wonder at the unified aetiology of the stromal corneal dystrophies, the validation of Knudson's hypothesis in retinoblastoma, and the bewildering array of gene defects underlying the retinal dystrophies. Even the complex field of glaucoma has at last begun to divulge some of its dark secrets.

Yet we must hope that these singular and rather separate strands will become woven into some sort of tapestry. The focus is broadening from the genes themselves to their function, their relations with the cellular environment, and to the wider biological implications of disease. Some of these areas have already begun to develop and promise to impact upon ophthalmology over the coming two decades.

The insights provided by molecular genetics have so far only yielded limited information about protein amount, activity, and function. As a consequence they have resulted in a resurfacing of interest in cell biology through which the role of specific genes and proteins in the maintenance of cellular, or tissue, function and phenotype may be revealed. Furthermore, the realisation that polygenic mechanisms underlie many disease processes renders imperative the understanding of protein-protein interactions in biological systems.

In general, all three levels of biological complexity-the cell, the organ, and the whole organism-must be investigated in order to provide insight into ocular physiology and disease as well as to validate new discoveries at the molecular level. Cell culture will continue to offer the advantages of simplicity and low expense while at the same time enabling the panorama of extracellular and intracellular events (for example, division, migration, translation, transcription, communication, signalling, intracellular enzyme cascades, and apoptosis) to be assessed. In order to investigate the three dimensional organisation and complexity of a tissue, organ culture may provide a more physiological view of cell-cell interactions. Also, however, animal models, in particular with the aid of transgenic techniques, will be essential to confirm the role for a gene or protein in a particular aspect of cell behaviour. Species variation and biological redundancy, however, ensure that ultimately human studies will provide the only conclusive evidence for the role for a given gene, or its encoded protein, in human ocular disease.

These methodologies have provided the foundations of our understanding of ocular physiology and disease and will continue to be essential components of our research armoury in the next millennium. In addition, the impact of bioinformatics - the application of computer technology to the management of biological information-upon emerging technologies should not be underestimated. Computers are already used to gather, store, analyse, and integrate biological and genetic information, which can then be applied to answer biological problems. As a consequence databases which contain genomic, cDNA, and protein sequences will provide an increasingly powerful tool for processing a vast range of information to predict gene and protein structure and function.

\section{Analysis of gene expression}

FUNCTIONAL GENOMICS

The entire three billion base pairs of the human genome will have been sequenced by the year 2003 and biological research will then enter the much heralded "postgenomic" era submerged under a morass of DNA sequence data. Within this will be the small proportion of sequence encoding our estimated 80 000-100 000 genes. Once these genes have been identified (no small task in itself) how can this information best be utilised? The term "functional genomics" has been coined to encompass the various challenges of discovering what regulates gene expression, what each of the gene's products (that is, RNA and protein) does, and how each interacts within a living organism.

A number of different strategies, including differential display and subtractive hybridisation, have been developed for studying cell specific or tissue specific gene expression. These have already been successfully used to identify many 
differentially expressed genes in a variety of ocular structures. These include ceruloplasmin, which is upregulated in the retina following optic nerve crush, ${ }^{1}$ respiratory and peptide processing genes, whose expression is modified by oxidative stress to lens cells, ${ }^{2}$ cyclin D1, which is important in retinal development, ${ }^{3}$ circadian clock genes, ${ }^{4}$ and metallothionein IIa, which is upregulated in lens epithelia from cataractous lenses. ${ }^{5}$

Since gene expression controls tissue structure and function, the ability to monitor simultaneously total cellular gene expression-representing perhaps thousands of genes or proteins-will allow the exploration of ocular physiology and pathology to enter a new dimension. This is now possible through new developments in nucleic acid arrays, which can be used to measure the expression patterns of thousands of genes. The idea of using such arrayed nucleic acid molecules to detect gene expression by hybridisation experiments is not new in itself. However, recent technological advances, in conjunction with the information from the DNA sequencing projects, open up exciting possibilities for obtaining large amounts of functional genetic information. ${ }^{67}$ This approach has been used in initial studies on the human retina and trabecular meshwork to demonstrate altered gene expression involving specific biochemical pathways in age related macular degeneration (AMD) and ocular hypertension respectively. ${ }^{8}$ Current high density microarrays carry thousands of individual cDNAs or synthesised oligonucleotides. However, in the near future it is predicted that chips will be produced that can array all of the 100000 human genes. Hybridisation using fluorescent probes could then allow the measurement of the assessment of total gene expression in a tissue sample at any one moment in time. The power of such technology as a tool in diagnosis, genetic analysis, and mutation detection is almost unimaginable. Further, as a result of its speed and sensitivity it will create many new targets for therapeutic intervention. ${ }^{10}$

\section{PROTEOMICS}

So far, the humble protein has seemingly been left out in the cold. Yet the advantages of analysing gene expression at a protein level are enormous since proteins are, after all, the functional molecules. Furthermore, analysis of mRNA may not truly reflect protein quantity and does not allow the analysis of post-translational modification. Proteomics is a novel research area which aims to provide a quantitative description of global protein expression and its changes under the influence of biological perturbations such as disease or drug treatment. ${ }^{11}$ The technique relies on the simultaneous separation of thousands of different proteins using high resolution two dimensional polyacrylamide gel electrophoresis (2D PAGE) and protein identification using mass spectrometry in conjunction with information from stored databases. Such databases containing digitally stored images of two dimensional electrophoretic patterns are already being established. These are likely to provide an important resource to compare, for example, differential expression patterns of proteins within normal and diseased tissue ${ }^{12}$ or their state of phosphorylation. ${ }^{13}$ The value of the technique is exemplified by its use in analysing the signal transduction pathways of the platelet derived growth factor $\beta$ receptor, ${ }^{14}$ where, following activation of the receptor, time dependent changes in the phorphorylation state of over 250 proteins were identified. The technique remains in its infancy in ophthalmic research but its importance was recently acknowledged at the 1999 ARVO and already databases are being developed for lens crystallins for future studies on cataract formation. ${ }^{15}$ Proteomics can confidently be predicted to be one of the major techniques of the next decade and will greatly enhance our understanding of biological changes associated with health and disease. Unfortunately, life is still more complicated than this. The activity and function of proteins is dependent on protein-protein interactions, an example being growth factor-receptor binding which elicits a downstream cascade of intracellular events. High throughput screening of protein-protein interactions can be undertaken using the yeast two hybrid system but recent developments in phage library technology may mean that these systems may be superseded. ${ }^{16}$

"WHOLE ORGANISM" INVESTIGATION

While novel techniques will allow the analysis of disease processes in vitro and facilitate the investigation of their broader impact at a single point in time, there remains the problem of following these processes longitudinally. Animal models of ophthalmic disorders, which have proved useful in the past, include models of retinal dystrophies (for example, the Abyssinian cat, RCS rat, and RDS mouse) and mouse models of cataractogenesis and anterior segment dysgenesis. More recent identification of the molecular defects in these models has also provided important information on the function of specific proteins. However, technologies allowing the development of novel model organisms have breathed new life into the investigation of the function of proteins. At present, the most definitive is to use transgenic technology and one of the most common approaches the knockout experiment, whereby the function of a specific gene is inactivated. An example is the mouse model of Norrie disease. The underlying gene was identified several years ago because of its $\mathrm{X}$ chromosomal location although its function remained unclear despite the definition of its sequence and of the likely structure of the encoded protein. ${ }^{17-19}$ Mutant mice which carry an amino acid substitution in the Norrie disease protein develop retrolental opacification and retinal disorganisation. ${ }^{20}$ Subsequent analysis of gene expression in association with the evolving phenotype of these mice has given valuable clues as to the gene's likely role in retinal vasculogenesis. ${ }^{21}$

The investigation of animal transgenic models has been extremely valuable for studying developmental defects such as aniridia, which is caused by mutations in the human paired box gene, $P A X 6{ }^{22}$ The Small eye mutant in mice is a developmental disorder caused by mutations within the murine homologue, Pax $6 .{ }^{23}$ Heterozygous mice have iris hypoplasia while homozygotes are anophthalmic. The human PAX6 gene, acting as a transgene in the Small eye mouse rescues the mutant phenotype. Interestingly, mice carrying multiple copies of the human gene also show specific developmental ocular abnormalities, suggesting that both reduced and increased levels of certain genes can cause developmental defects-a clear lesson for future attempts at gene therapy. ${ }^{24}$

The potential of such animal models for the development and definition of novel putative treatment regimens should not be underestimated. One such example is the mouse model of gyrate atrophy. In humans, ornithine delta-aminotransferase (OAT) deficiency causes gyrate atrophy, a rare autosomal recessive chorioretinal degeneration. ${ }^{25}$ In the mouse knockout young animals post-weaning develop a retinal degeneration apparently analogous to human gyrate atrophy. ${ }^{26}$ It has long been suggested that dietary manipulation by means of a low arginine diet might reduce the long term effects of OAT deficiency but evidence for this in humans has remained limited as the rigours of a low arginine diet are enormous. ${ }^{27}$ The ability to test such theories on mice is a great advance and the implications of successful dietary therapy, which have indeed been demonstrated in a preliminary form, are self 
evident. ${ }^{28}$ Further mouse models will be equally helpful in testing many other forms of therapeutic intervention.

\section{Future therapeutic approaches}

CONVENTIONAL PHARMACOLOGICAL INTERVENTION

Conventional ocular pharmacology will continue to be a major feature in treatment, certainly in the early part of the next century. The management of primary open angle glaucoma (POAG), for example, will still rely upon the current array of drugs. However, at the present time a major block in the development of new and more effective therapies for heterogeneous groups of disorders such as POAG (as well as perhaps AMD and retinitis pigmentosa) is our inability to identify true subforms of the disease. In the absence of effective subclassification, treatment regimens are assayed on mixed groups of patients and their true efficacy is lost in the communal melting pot. For POAG the chromosomal localisation of several genetic forms has already been defined ${ }^{29}$ and in the future better subclassification of POAG, perhaps based upon different genetic aetiologies, will allow treatments to be more accurately and effectively targeted.

We will see an improvement in delivery techniques with emphasis on site specific drug delivery and long term action of pharmacological agents. ${ }^{30}$ Drug containing liposomes have been designed with a targeting ligand to allow preferential binding to its target tissue. ${ }^{31}{ }^{32}$ This can extend the intravitreal half life of drugs for conditions such as viral retinitis. ${ }^{33}$ Toxins or drugs conjugated with ligands for cell receptors or monoclonal antibodies offer excellent site specific delivery. For example, ricin conjugated to either transferrin or to an antibody can be targeted to prevent proliferative vitreoretinopathy ${ }^{34}$ and posterior capsule opacification. ${ }^{35}{ }^{36}$ Site directed photosensitisers, which have previously been used to target dermal conditions, are now being employed to treat ocular neovascularisation. Indeed, photodynamic therapy is currently showing promising outcomes for the obliteration of the neovascular membranes associated with AMD. ${ }^{37}$

With our ever increasing knowledge of the regulators of cell function, intracellular signalling cascades, and transcription controls we now have the ability to pinpoint and modulate a single biomolecular feature within a particular cell type or tissue. While this approach is still in its infancy it will make a major impact in the coming decades. Exogenous transforming growth factor $\beta$ (TGF $\beta$ ) has already been shown to promote corneal and conjunctival scarring ${ }^{38}$ while application of vascular endothelial growth factor (VEGF) promotes ocular angiogenesis. ${ }^{39}$ Conversely, pharmaceutical inhibition of these molecules-for example, using neutralising antibodies, may enable the prevention corneal scarring, ${ }^{40}$ bleb failure in glaucoma surgery, ${ }^{41}$ and angiogenic responses in the eye. ${ }^{42} \mathrm{With}$ the advent of recombinant technology the use of antibodies is becoming more acceptable since human antibodies or antibody fragments, hopefully without inherent toxicity, can be generated by ribosome or phage display. ${ }^{43}{ }^{44}$ These approaches are now in various stages of clinical trials and may become an important adjuvant to the armoury of the ophthalmologist. Many other therapeutic avenues are being explored. These include the use of soluble receptors to mop up specific ligands, inhibition of receptor kinases, ${ }^{45}$ and competitive inhibition using amino acid sequences (for example, blocking cell matrix interaction with RGD based peptides). In addition, the application of natural inhibitory proteins, such as angiostatin and endostatin, which reduce angiogenesis and anti-apoptotic agents, such as neurotrophic factors and caspase inhibitors, are being investigated. ${ }^{46}$ As molecular genetics and proteomics identify other important cellular regulators we will be able to develop new strategies for controlling cell and tissue behaviour.

\section{GENE THERAPY}

In the past decade there has been a huge expansion of our attempts to develop gene therapy. To date more than 350 clinical trials have been approved. This period has also seen extensive investment into optimising conditions for delivery of the "medicine" for defective genes. A variety of techniques attempt to insert a functioning gene into cells directly either to correct a genetic error or to express a novel protein which is secreted into the local environment. Alternatively, other technologies attempt to alter mRNA species. These include the application of antisense oligonucleotides to hybridise with mRNA in order to block its translation, and the use of transfer vectors encoding catalytic RNA (ribozymes) which degrade mRNA or lead to activation of RNAse $\mathrm{H}$ which in turn degrades RNA/RNA hybrids. ${ }^{48}{ }^{49}$ Further developments in this area include the introduction of a cDNA, which encodes an antisense mRNA, which will bind to a specific RNA and lead to the degradation of the RNA/RNA hybrids.

Despite all the hype there is still little conclusive evidence that a gene therapy protocol has been successful in the treatment of human disease.$^{50}$ However, great potential remains. Gene transfer in animal models has successfully delayed (but not prevented) photoreceptor death in a variety of inherited retinal degenerations, ${ }^{51-55}$ has shown restoration of normal lysosomal function in mucopolysaccharidosis, ${ }^{56}$ and has introduced suicide genes into proliferative ocular diseases such as proliferative vitreoretinopathy ${ }^{57}$ and posterior capsular opacification. ${ }^{58}$ While it is clear that gene therapy may offer a valuable and attractive approach to many ocular diseases many aspects remain to be successfully addressed as we proceed into, and beyond, the millennium. These include improvement of vector design, achieving site directed expression, and improvement in the stability and maintenance of transfected gene expression.

The molecular revolution has transformed our understanding of all aspects of medicine at a pace that could scarcely have been predicted a decade ago. Our ability to dissect the molecular mechanisms involved in a multitude of disease processes now reaches not only inherited developmental conditions and complex or polygenic conditions but also the mechanisms underlying the response to infection, inflammation, trauma, and even surgery. The future is (almost) upon us. And who knows? Perhaps by the year 2019 , a new breed, the molecular pharmacogeneticist, will be receiving referrals from general ophthalmic physicians to give advice on appropriate and novel management regimens ....

GB and PB are Wellcome Trust clinician scientist fellows.

G C M BLACK

University Department of Ophthalmology, Manchester Royal Eye Hospital, Manchester M13 9WH and University Department of Medical Genetics and Regional Genetics Service, St Mary's Hospital, Manchester M13 0JH

M E BOULTON

Cell and Molecular Biology Unit, Department of Optometry and Vision Sciences, Cardiff University, Cardiff CF10 3YJ

P N BISHOP

University Department of Ophthalmology, Manchester Royal Eye Hospital, Manchester M13 9WH and Wellcome Trust Centre for Cell-Matrix Research, School of Biological Sciences, University of Manchester, M13 9JH

D MCLEOD

University Department of Ophthalmology, Manchester Royal Eye Hospital, Manchester M13 9WH 
Correspondence to: Mr G C M Black, Regional Genetic Service, Central Manchester Healthcare NHS Trust, St Mary's Hospital, Manchester M13 0JH

1 Levin L, Geszvain K. Expression of ceruloplasmin in the retina: induction after optic nerve crush. Invest Ophthalmol Vis Sci 1998;39:157-63.

2 Carper D, Sun J, Iwata T, et al. Oxidative stress induces differential gene expression in a human lens epithelial cell line. Invest Ophthalmol Vis Sci 1999;40:400-6.

3 Godbout R, Andison R. Elevated levels of cyclin D1 mRNA in the undifferentiated chick retina. Gene 1996;182:111-15.

4 Green C, Besharse J. Identification of a novel vertebrate circadian clock-regulated gene encoding the protein nocturnin. Proc Natl Acad Sci USA 1996;93:14308-9.

5 Kantorow M, Kays T, Horwitz J, et al. Differential display detects altered gene expression between cataractous and normal human lenses. Invest Ophthalmol Vis Sci 1998;39:2344-54.

6 Schena M, Heller RA, Thieriault TP, et al. Microarrays: biotechnology's Schena M, Heller RA, Thieriault TP, et al. Microarrays: biotechnology
discovery platform for functional genomics. TITECH 1998;16:301-6.

discovery platform for functional genomics. TITECH 1998;16:3
7 Lander ES. Array of hope. Nature Genet (Suppl) 1999;21:3-4.

8 Bernstein SL, Somiari RI, Robinson G, et al. Rapid analysis of gene expression differences between ARMD and normal retina. Invest Ophthalmol Vis sion differences betw

9 Gonzales P, Rowlette LLS, Borrás T. Genes upregulated in the human trabecular meshwork under high intraocular pressure. Differential expression analysis by gene array. Invest Ophthalmol Vis Sci 1999;40:s505.

10 Debouck C, Goodfellow P. DNA microarrays in drug discovery and development. Nat Genet 1999;21:48-50.

11 Anderson NL, Anderson NG. Proteome and proteomics: new technologies, new concepts, and new words. Electrophoresis 1998;19:1853-61.

12 Celis JE, Østergaard M, Jensen NA, et al. Human and mouse proteomic databases: novel resources in the protein universe. FEBS Lett 1998;430:6472 .

13 Blackstock W, Weir M. Proteomics: quantitative and physical mapping of cellular proteins. Trends Biotechnol 1999;17:121-7.

14 Soskie V, Gorlach M, Poznanovic S, et al. Functional proteomics analysis of signal transduction pathways of platelet-derived growth factor beta signal transduction pathways of plat
receptor. Biochemistry 1999;38:1757-64.

15 David LL, Lampi KJ. Rapid mapping of crystallin identities on two-dimensional electrophoresis gels by mass spectrometry. Invest Ophthaltwo-dimensional electrop.
mol Vis Sci 1999;40:s969.

16 Borrebaeck CAK. Tapping the potential of molecular libraries in functional genomics. Immunol Today 1998;19:524-7.

17 Chen ZY, Hendriks RW, Jobling MA, et al. Isolation and characterization of a candidate gene for Norrie disease. Nat Genet 1992;1:204-8.

18 Berger W, Meindl A, van de Pol TJ, et al. Isolation of a candidate gene for Norrie disease by positional cloning. Nat Genet 1992 1:199-203.

19 Meitinger T, Meindl A, Bork P, et al. Molecular modelling of the Norrie disease protein predicts a cystine knot growth factor tertiary structure. Nat Genet 1993 5:376-80.

20 Berger W, van de Pol D, Bachner D, et al. An animal model for Norrie disease (ND): gene targeting of the mouse ND gene. Hum Mol Genet 1996;5: 51-9.

21 Richter M, Gottanka J, May CA, et al. Retinal vasculature changes in Norrie disease mice. Invest Ophthalmol Vis Sci 1998;39:2450-7.

22 Hanson I, Churchill A, Love J, et al. Missense mutations in the most ancient residues of the PAX6 paired domain underlie a spectrum of human residues of the PAX6 paired domain underlie a spectrum of
congenital eye malformations. Hum Molec Genet 1999;8:165-72.

23 Hill RE, Favor J, Hogan BLM, et al. Mouse small eye results from mutations in a paired-like homeobox-containing gene. Nature 1991;354:522-5.

24 Schedl A, Ross A, Lee M, et al. Influence of PAX6 gene dosage on development: overexpression causes severe eye abnormalities. Cell 1996;86: $71-82$.

25 Ramesh V, Benoit LA, Crawford P, et al. The ornithine aminotransferase (OAT) locus: analysis of RFLPs in gyrate atrophy. Am f Hum Genet 1988; 42:365-72.

26 Wang T, Lawler AM, Steel G, et al. Mice lacking ornithine-delta-aminotransferase have paradoxical neonatal hypoornithinaemia and retinal degeneration. Nat Genet 1995;11:185-90.

27 Kaiser-Kupfer, MI, de Monasterio FM, Valle D, et al. Gyrate atrophy of the choroid and retina: improved visual function following reduction of plasma ornithine by diet. Science 1980;210:1128-31.

28 Wang T, Milam AH, Valle D. Correction of ornithine acumulation prevents retinal degeneration in a mouse model of gyrate atrophy of the choroid and retina. Am f Hum Genet 1996;59(Suppl):70.
29 Sarfarazi M, Child A, Stoilova D, et al. Localization of the fourth locus (GLC1E) for adult-onset primary open-angle glaucoma to the 10p15-p14 (1998;62:641-52.

30 Bourlais C, Acar L, Zia H, et al. Ophthalmic drug delivery systems-recent advances. Progr Ret Res 1998:17:33-58.

31 Ranade V, Hollinger M. Drug delivery systems. New York, London, Tokyo: CRC Press, 1996.

32 Velpandian T, Gupta S, Gupta Y, et al. Ocular drug targeting by liposomes and their corneal interactions. F Microencapsulation 1999;16:243-50.

33 Kuppermann BD, Assil KK, Vuong C, et al. Liposome-encapsulated (S)-1(3-hydroxy-2-phosphonylmethoxypropyl)cytosine for long-acting therapy of viral retinitis. F Infect Dis 1996;173:18-23.

34 Handa J, Keithahn M, Jaffe G. Transferrin-ricin A chain toxin limits the development of experimental vitreoretinopathy. Exp Eye Res 1996;62:68996.

35 Goins K, Ortiz J, Fulcher S, et al. Inhibition of proliferating lens epithelium with anti-transferrin receptor immunotoxin. 7 Cataract Refract Surg 1994;20:513-16.

36 Clark D, Emery J, Munsell M. Inhibition of posterior capsule opacification with an immunotoxin specific for lens epithelial cells: 24 month clinical results. F Cataract Refract Surg 1998;24:1614-20.

37 Chong N, Bird A. Alternative therapies in exudative age related macular degeneration. Br 7 Ophthalmol 1998;82:1441-3.

38 Mita T, Yamashita H, Kaji Y, et al. Functional difference of TGF $\beta$ isoforms regulating corneal wound healing after excimer laser keratectomy. Exp Eye Res 1999;68:513-19.

39 Aiello L. Vascular endothelial growth factor and the eye. Biochemical mechanism of action and implications of novel therapies. Ophthalmic Res 1997;29:354-62.

40 Moller-Pedersen T, Cavanagh $\mathrm{H}$, Petroll W, et al. Neutralizing antibody to TGF $\beta$ modulates stromal fibrosis but not regression of photoablative effect following PRK. Curr Eye Res 1998;17:736-47.

41 Cordeiro M, Gay J, Khaw P. Human anti-TGF- $\beta$ monoclonal antibody: a new antiscarring agent

42 Adamis A, Shima D, Tolentino M, et al. Inhibition of VEGF prevents ocular neovascularisation in a non-human primate. Arch Ophthalmol 1996;114: 66-71.

43 Dall'Acqua W, Carter P. Antibody engineering. Curr Opin Struct Biol 1998;

44 Vaughan T, Osbourn J, Tempest P. Human antibodies by design. Nat Biotechnol 1998;16:535-9.

45 Bergers G, Javaherian K, Lo K, et al. Effects of angiogenesis inhibitors on multistage carcinogenesis in mice. Science 1999;284:808-12.

46 DiPolo A, Aigner L, Dunn R, et al. Prolonged delivery of brain-derived neurotrophic factor by adenovirus infected Muller cells temporarily rescues injured retinal ganglion cells. Proc Natl Acad Sci USA 1998;95:3978-83.

47 Salvesen G. Programmed cell death and caspases. APMIS 1999;107:73-9.

48 Rossi J. Ribozymes, genomics and therapeutics. Chemistry and Biology 1999; 6:R33-7.

49 James H, Gibson I. The therapeutic potential of ribozymes. Blood 1999;91: $371-82$

50 Anderson W. Human gene therapy. Nature 1998;392:25-30.

51 Bennett J, Tanabe T, Sun D, et al. Photoreceptor cell rescue in retinal degeneration (rd) mice by in vivo gene therapy. Nat Med 1996;2:649-54.

52 Bennett J, Zeng Y, Bajwa R, et al. Adenovirus-mediated delivery of rhodopsin-promoted bcl-2 results in a delay in photoreceptor cell death in the $\mathrm{rd} / \mathrm{rd}$ mouse. Gene Ther 1998;5:1156-64.

53 Jomary C, Vincent K, Grist J, et al. Rescue of photoreceptor function by AAV-mediated gene transfer in a mouse model of inherited retinal degenAAV-mediated gene transfer in a mou

54 Cayouette M, Gravel C. Adenovirus-mediated gene transfer of ciliary neutrophic factor can prevent photoreceptor degeneration in the retinal degeneration (rd) mouse. Hum Gene Ther 1997;8:423-30.

55 Chen J, Flannery J, LaVail M, et al. Bcl-2 overexpression reduces apoptotic photoreceptor cell death in three different retinal degenerations. Proc Natl Acad Sci USA 1996;93:7042-7.

$56 \mathrm{Li} \mathrm{T}$, Davidson BL. Phenotype correction in retinal pigment epithelium in murine mucopolysaccharidosis VII by adenovirus-mediated gene transfer. Proc Natl Acad Sci USA 1995;92:7700-4.

57 Murata T, Kimura H, Sakamoto T, et al. Ocular gene therapy: experimental studies and clinical possibilities. Ophthalmic Res 1997;29:242-51.

58 Malecaze F, Couderc B, Deneuville S, et al. Suicide gene transduction: feasibility in lens epithelium and in prevention of posterior capsule opacification in rabbits. Invest Ophthalmol Vis Sci 1999;40:S197. 